\title{
Grounding Privacy in Mediated Communication
}

\author{
Natalia A. Romero \\ Industrial Design Engineering Faculty, Delft University of Technology \\ Panos Markopoulos \\ Industrial Design Department, Eindhoven University of Technology \\ Saul Greenberg \\ Department of Computer Science, University of Calgary
}

\begin{abstract}
This paper addresses interpersonal privacy coordination in the context of mediated communication, emphasizing the dialectic and dynamic nature of privacy. We contribute the Privacy Grounding Model - built upon the Common Ground theory - that describes how connected individuals create and adapt privacy borders dynamically and in a collaborative process. We present the theoretical foundations of the model. We also show the applicability of the model, where we give evidence from two case studies that illustrate how it can describe privacy coordination among users of an instant messaging application and a desktop awareness system. More generally, we believe designers can use the Privacy Grounding Model to reflect on how their system supports or fails to support people's lightweight privacy coordination mechanisms, and in particular how communicators within the system create and use privacy border representations as common ground. Finally we briefly consider the design of interactive and lightweight privacy grounding mechanisms.
\end{abstract}

\section{Introduction}

The broad adoption of Internet, mobile phones and - especially in the last decade - social networking applications has made individuals in developed societies almost continuously connected and accessible to others. As a result, people now expect others to be available and responsive for communication. This 'always available' shift in culture often places individuals under considerable social and organizational pressure.

By means of sustained and almost continuous connectivity, users of communication media acquire and maintain awareness of others. For the purposes of this paper, awareness is an understanding of the whereabouts of others, or their activities, feelings, experiences, or - more generally - their current status. Conversely, individuals provide to their social network, either through explicit action or through automated technical means, rich and frequent information about themselves. Such information can come in many forms, including: online status in instant messaging applications; regular postings to social networks; blog postings and feeds; captured real-time audio and video of oneself (e.g., as in media spaces); and contextual information gathered from sensors, such as one's location.

On the one hand (and regardless of the communication medium), others can translate this awareness information into fine-tuned opportunities and expectations of availability, that can enable opportunistic and timely social interaction that many truly enjoy or value for their utility. On the other hand, regularly sharing this information makes individuals

Cite as:

Romero, N.A., Markopoulos, P. and Greenberg, S. Grounding Privacy in Mediated Communication. Report 2011-999-11, Department of Computer Science, University of Calgary, Calgary, Alberta, Canada T1W $1 \mathrm{~S} 2$. 
accountable for their availability: it can compromise their prerogative to choose whether and when to engage in communication. As a result plausible deniability is severely diminished (Nardi, Whittaker and Bradner, 2000) as technology hinders an individual's ability to conceal related choices and social behaviour that had been possible over traditional media. Thus individuals may feel coerced to accept undesired interruptions, which are experienced as privacy intrusions. Arguably, this trade-off between connectivity and awareness makes interpersonal privacy a primary design concern in the design of related technologies.

In the non-technological world, people manage their availability for social interaction and the information about themselves that they make available to others by selfregulating their interaction behaviours. Interpersonal aspects of privacy regulation are governed by existing social norms and build upon people's social skills (Erickson et al. 1999). Palen and Dourish (2003) argue that individuals are challenged by modern technology, where they find it difficult to regulate and protect themselves (and others) from undesired interaction. This is a well known challenge in the design and study of interpersonal awareness systems. Some examples illustrate this point.

The ASTRA study (Markopoulos et al., 2004; Romero et al., 2005; Romero et al., 2007a) identified privacy interaction breakdowns in which too much or too little information resulted in an undesired state of interaction. It showed how such undesired states are associated with affective costs. These include feelings of being obliged to react to a message, or feelings of uncertainty and disappointment towards unresponsive interaction.

Patil and Kobsa (2004) reported peoples' privacy concerns in instant messaging regarding availability and interruptions. People were found to resort to different strategies to keep their interaction at desired levels by controlling how they appeared to others. Their study participants reported availability disclosure as an important mechanism to regulate interruptions and distractions from current tasks.

McFarlane (2002) studied coordination of interruptions in computer-based multitasking. When discussing how systems should support mediated interruptions, he concluded that people should be given mechanisms that allow them to both assess and announce interruption moments. These results were confirmed and extended in an experiment by Romero et al. (2007b): when communicators shared a common goal, they were more likely to assess recipient's availability and time-pressure before initiating an interruption. Where the system shielded recipients from interruptions, they developed strategies to manually overcome such automatic protection in order to collaborate with the interrupter.

In seeming contrast, field tests of experimental ubiquitous systems have repeatedly failed to reveal any 'privacy concerns' perhaps suggesting that such technologies are innocuous and easily accepted (e.g., Miller and Stasko, 2001; Metaxas et al. 2007). Yet looking closer at these studies, and where clarifications are provided, it becomes clear that privacy is implicitly equated to undesired access to information about oneself, and often considered only globally at the level of the overall experience from the field study rather than within a finer time frame. Furthermore, such evaluations are done in the safe context of a research study, with a well defined social context (e.g., fixed information content and recipients) and for short periods of time. This, of course, influences 
subjective reports regarding privacy and even privacy related behaviours (Garde Perik et al. 2008).

The difficulty of optimizing the right trade-off between awareness and privacy relates to the dynamic and dialectic nature of interpersonal privacy. This tradeoff was described previously by Altman (1974) and later echoed by Palen and Dourish (2003). While this is slowly gaining broader acceptance by researchers in human computer interaction, interaction design rarely reflects the fact that privacy preferences are constantly under negotiation and, as argued by Petronio (2001), that privacy borders are set cooperatively by the interacting individuals.

Our research contributes a theory that articulates how connected individuals create and adapt privacy borders both dynamically and in a collaborative process. Our goal is to provide a theoretical foundation for related discussions that will allow not only the classification and explanation of interpersonal privacy behaviours of individuals in a communication setting, but also to inform the design of related systems.

The remainder of this article is structured as follows. First, we provide an overview of related efforts that examine privacy regulation in modern communication media. We then discuss the nature of interpersonal privacy phenomena surrounding mediated communication, where we build on the theories of Altman (1975) and Petronio (2001). Next, the Privacy Grounding Model (PGM) is introduced as an adaptation of the Common Ground theory (Clark, 1996), where it describes the coordination process of interpersonal privacy borders in mediated communication. Subsequently, we report two empirical studies that helped shape and validate the PGM. We conclude with a discussion on the findings, design implications, and future work.

Our overarching argument is that designing for privacy is about designing systems that let communicators efficiently and effectively perform their respective parts of the process of initiating/reacting to communication. The Privacy Grounding Model (PGM) that we contribute describes this coordination process of interpersonal privacy borders in mediated communication.

\section{Related Work}

\subsection{Towards interactive solutions for interpersonal privacy management}

Traditionally. privacy in relation to computing and communication systems has been conflated with issues of access to personal information. Dourish (1993), for example, argued that users of mediated communication systems are primarily concerned with privacy issues regarding their interaction with others, such as protecting personal space from undesired access. This is a somewhat narrow view of privacy, and several researchers have since refined and broadened this view beyond access.

Palen and Dourish (2003) provided a first conceptual framework of interpersonal privacy in mediated settings. Contrasting information security and confidentiality perspectives on privacy, they introduced the concept of genres of disclosure arguing that limited disclosure can be employed by individuals to limit accessibility for communication. This concept drew attention to the interplay of technology characteristics and an individual's privacy intentions and how appropriate social expectations and social practices are produced. It provided a first understanding of privacy concerns regarding 
technology, but does not shed light into the process of communicating privacy intentions itself when disclosing awareness.

Bellotti and Sellen (1993) studied privacy in the context of media spaces, which are networked environments that support sustained audio and video connections between members of a group. Their design framework addresses privacy as a social construct and as a highly subjective and dynamic process (Harper et al., 1992), where privacy related behaviours are highly contingent upon technology. For example, increasing the resolution or frequency of displaying video information might alter one's earlier preferences to constantly display visual presence to others. As a result, Bellotti and Sellen's framework argued in favour of control and feedback mechanisms to support the subjective and dynamic process of information disclosure. The framework provides users of media spaces with mechanisms to make informed decisions of how to control the capturing, processing, accessing and purpose of use of one's personal information. However, the authors observed that it was technically unfeasible that systems could provide feedback on purpose of use. Therefore, users are forced to control 'purpose of use' outside the system based on social knowledge and culture..

Continuing on related interaction perspectives on privacy, Nardi, Whittaker, and Bradner (2000) emphasized the importance of supporting users of mediated communication coordinate the process of their interaction. The authors introduced the concept of outeraction, defined as lightweight mechanisms outside the information exchange that individuals develop and use to reach out to others in patently social ways. However, their accounts on outeraction mechanisms is limited to a specific set of the technology aspects observed in Instant Messaging.

\subsection{Automated Interruption Management}

Every initiation of communication can be framed as a potential interruption. Under this framing, the topic interruption management has been studied extensively. Two opposing approaches can be schematically distinguished: an automated vs. a manual approach.

In the automated approach, the system takes the role of an interruption mediator brokering or filtering interruption attempts. For example, the Personal Reachability Management Systems by Reichenbach et al. (1997) offered automated availability management aimed at minimizing recipients' effort when dealing with undesired communicative attempts. Processing an interruption request was based on matching what the initiator has specified as the context of the communication attempt and what the recipient has defined as criteria for accepting an interaction request. Similarly, the Personal-Level Routing (Roussopoulos et al., 1999) served as a personal proxy to automatically maintain person-to-person reachability. The system offered a routing service aimed at protecting users from undesired contacts tracking their location, filtering incoming messages and forwarding them to the appropriate communication medium according to a user's personal privacy rules and location.

A drawback of such automated solutions is that a special effort and premature commitment is required from users, as they have to set privacy rules explicitly and a priori. Empirical evidence on the use of customized software (Mackay, 1999) and the groupware calendar system (Palen, 1999) showed that users are unlikely to configure systems to manage their privacy in this way. In most cases, default settings are adopted. Lederer, Hong, and Landay (2004) argued that users are often not comfortable setting 
preferences unless they have suffered a privacy breakdown. In their evaluation of Faces (Lederer et al., 2004), a prototype interface for managing personal privacy in ubiquitous computing settings, the authors report that test participants set privacy preferences differently than their a priori stated preferences and had trouble recalling their current settings which hampered their ability to predict a disclosure throughout the experiment. This is perhaps unsurprising as a discrepancy between attitudes and behaviour in the domain of privacy, something that has been discussed often before (van de Garde-Perik, 2008), but also portrays the difficulty of deciding upon privacy preferences outside a concretely specified context. The authors argued that excessive configuration of privacy rules should be avoided; instead designers: "should enable users to manage privacy as a natural consequence of their primary actions involving the system" (Lederer, et al., 2004). To our knowledge, related literature has still not put forward interactive solutions that will fulfil this requirement.

\subsection{Manual Interruption Management}

Solutions offering interactive interruption management provide people with mechanisms to self-manage their privacy. This, of course, needs to be done at low cost to the end-user.

Pursuing the interactive approach, Woodruff and Aoki (2003) argued for very low effort and low commitment approach to establishing communication. They evaluated a half duplex audio communication appliance that supported a Push-to-Talk initiation of communication. This mechanism was effortless and flexible enough to allow users to implement a set of interaction strategies to coordinate their availability including the maintenance of plausible deniability, delays or omissions in responding to communication acts by others. Such mechanisms created a sense of reduced interactional commitment between users supporting a lightweight interactive negotiation process. However, it appears from their reported data that the system failed to support more complex scenarios where tensions could be more visible between participants. Similarly, Wiberg and Whittaker (2005) used their Negotiator system to show how manual availability management might create social tension for the interacting parties and incur cognitive costs when conflicts occur.

Common to related works is that any mechanism that supports privacy - whether automatic or manual, or for regulating information or interaction - must be lightweight if it is to be used, and must align with established and emerging social practices in the coordination of interpersonal privacy.

\section{Privacy as Border Regulation}

The previous findings of privacy largely result from evaluations of systems in use. We now turn to theories of privacy, which provides a more general and perhaps more comprehensive perspective on privacy. In particular, we build upon Altman's theory, as it has exerted substantial influence in how researchers in the field of human computer interaction and mediated communication view privacy issues surrounding networked applications, e.g., as used by Dourish (1993).

Altman (1975) defined privacy as a border regulation process where individuals use mechanisms to open or close their borders to regulate if and how interaction takes place within their environment. Altman's definition acknowledges that individuals modify and 


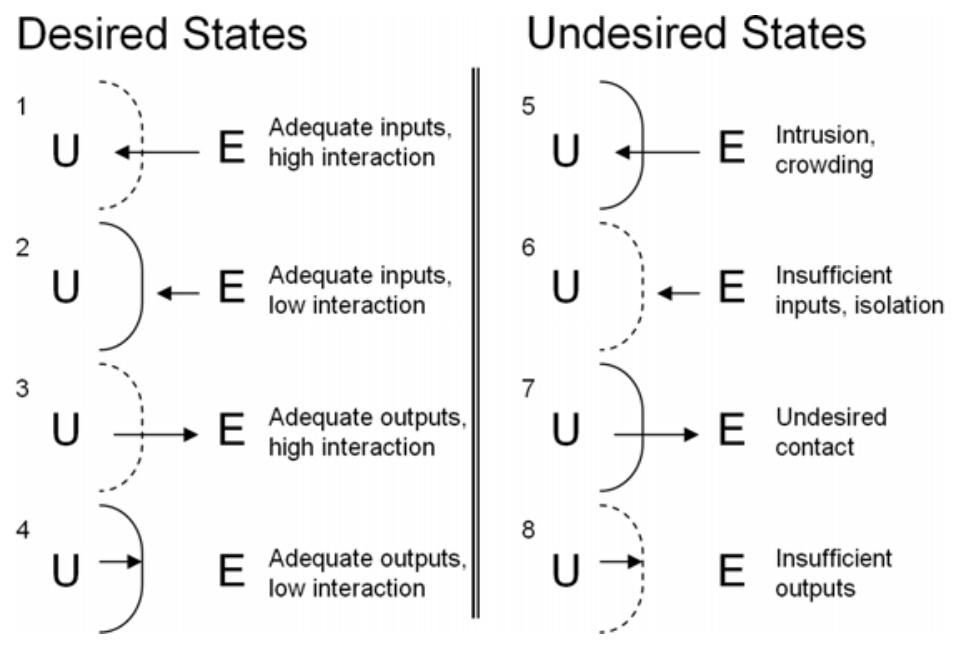

Figure 1. Altman's model of privacy borders regulation. A continuous line represents an individual's border $(U)$ that is closed to the environment $(E)$. A dashed line represents an opened border. The arrows represent initiation of interaction by the individual or the environment. To the left are 4 desired states of interaction between individual and environment; to the right are 4 undesired states of interaction

continuously re-assess their borders in response to stimuli by their environment and their own needs for social interaction. Altman argued that a failure to achieve the desired privacy state by means of border regulation could cause negative effects. For example, an open border that was never crossed could cause an undesired feeling of isolation, while a closed border that was crossed could violate the wish to be alone and creates a feeling of disrespect.

Figure 1 shows how this boundary regulation process can result in desired and undesired states of interaction with the environment. The figure uses a continuous line to represent an individual's border that is closed to the environment (preventing any interaction) and a dashed line when the border is opened (allowing interaction to take place). When a person attempts to initiate an interaction with someone in the environment, this is shown as an arrow pointing from the person (U) to the environment (E) and vice versa. The diagrams show the possible cases of desired and undesired outcomes for an attempt to interact. For example, the desired state represented in (1) illustrates situations such as when a person opens the door and lets someone else in, while in (4) a person is glancing away and avoiding unwanted conversation with someone. From the undesired scenarios, (6) illustrates situations such as when a person invites someone (E) for communication, but (E) does not engage. Alternatively, in (7) a person is hoping to avoid (E), but had to spend an evening together.

Altman's perspective on privacy acknowledges but leaves implicit interpersonal interactions that are crucial in mediated settings: privacy borders are not set unilaterally by an individual but are regulated by both communicating parties pursuing their individual needs and responding to the needs for the other. Such a broader perspective is advocated by Petronio (2001), who extended Altman's theory by introducing the element of collaboration in privacy regulation: both sender and receiver are mutually responsible over the information exchanged. Petronio's framework encompasses the perspective of both communicating parties, describing how the parties develop and use rules to agree on whether to disclose certain information, and on which basis each party takes 
responsibility over the information disclosed. Petronio also acknowledged the importance of understanding other signs (outside the exchange of information), i.e., to interpret cues of when is the right time to disclose something, to whom, how, and so on. She recognized that this "implicit" information seems to be crucial for the disclosure decision.

Bellotti and Sellen (1993), in their validation of the privacy framework mentioned earlier, also observed the need to communicate such parallel signs. Most of the systems they evaluated were technically unable to support control and feedback behaviours related to intentions of use. Instead, control mechanisms seemed to be primarily focused on helping users set their preferences for disclosing information rather than supporting how they would coordinate with others what to disclose and for what purposes. Bellotti and Sellen expected that people would use social rules to coordinate such purposes, but they did not describe how a system could support such rules.

Boyle and Greenberg (2005) developed a privacy vocabulary that builds upon the theoretical work of Altman and others, where they precisely define and describe the nature of the privacy concerns in the context of interpersonal coordination needs (see also Boyle, Neustaedter and Greenberg, 2009). At a high level, they describe the process of privacy regulation by means of solitude, autonomy and confidentiality controls. These concepts refer to people's needs to control desired level of interaction (availability), to identify one's behaviours within the community (whether collaboratively or individualistic), and to control the access to personal information (fidelity, accuracy) respectively.

In conclusion, related work has argued for the collaborative and dynamic nature of privacy border management, especially for managing interpersonal privacy in mediated communication. However, there is as yet no account of these mechanisms nor a theoretically motivated understanding of how best to design interactive controls for related systems. To address this apparent gap we turn to Clark's (1996) theory of Common Ground.

\section{Common Ground Theory}

Common Ground (Clark, 1996) is a theory of language use that emphasizes the dynamic collaborative process that people engage to develop the necessary common ground for the success of their communication. According to Clark, people develop common ground to communicate meanings to each other efficiently; in turn communication helps develop this common ground further.

Common Ground theory was originally conceived to describe human behaviours in face-to-face communication, but has been successfully used to make predictions and inform the design of system mediated communications. For the purposes of modelling privacy coordination in mediated settings, only a subset of the components and principles of Common Ground will be used. These elements are introduced briefly below; readers wishing to read more can refer to Clark's own book (Clark, 1996) for an extensive exposition or to the succinct tutorial chapter by Monk (Monk, 2003).

Three key concepts of Common Ground theory will be applied to interpersonal privacy coordination: collaboration, signalling, and grounding. The theory describes the collaborative process of developing common ground by signalling where communicators collaborate to represent their intentions and grounding; the goal is to collaboratively construct a shared meaning of such intentions. 
Collaboration models coordination activities as contribution pairs, involving presentations and reactions by which communicators try to reach closure for each signal used to communicate. An example is the following initiation-reaction pair: 'hi, coffee?' 'sure, give me a moment'. Initiators contribute with presentations of their intentions to interact and recipients contribute with reactions to communicate their understanding of those intentions. These paired contributions are ruled by the principle of least collaborative effort: communicators in a joint activity try to express no more than what they perceive as sufficient for advancing the current communication.

Signalling describes the use of at least two tracks to communicate both the content of communication (track-I signals) and the means to coordinate the process of communication (track-II signals). With every presentation, contributors produce track-II signals projecting evidence of understanding that they consider to be valid, economical, and timely enough for current purposes. They are in the background and brief as they have to be undemanding in their presentation to allow track-I signals to be more prominent. They are simultaneous to track-I signals so the coordination can occur at the same time as the content is presented, and at the same time they are distinctive enough from track-I signals to easily understand their coordination purposes. These four properties - background, brief, distinctive, and simultaneous - ensure that the coordination process is lightweight.

Grounding is an important mechanism to ensure that the coordination process proceeds effectively. Grounding is about shared understanding. It describes the use of existing representations of common ground (i.e., of the shared understanding), or in developing new common ground to ensure the correct understanding of a track-II signal. Communicators need such common ground representations if they are to correctly establish a shared understanding of their intentions to communicate.

Clark identified three basic common ground representations. First, conventions represent any custom or practice that could be associated with the use of a communication protocol. For example, conventions often define the way communicators interact with each other, e.g. turn taking, acknowledging receipt, waiting for someone to finish, etc. External representations characterize any external demonstration of track-II signals, which all communicators can see. These include pointing, indicating, demonstrating, etc. Shared previous events represent any information related to the coordination of previous events that have been shared by all communicators.

\section{Privacy Grounding Model}

We now introduce our own Privacy Grounding Model (PGM), a descriptive model which provides a generic characterization of the social practices that describe interpersonal privacy coordination activities in mediated settings (Romero and Markopoulos, 2009). Our model assumes that privacy needs and preferences derive and evolve in a fluid way during the course of the interaction between people. We build upon prior work to develop this model. In particular, we use the three elements of the Common Ground theory introduced above: collaboration, signalling and grounding. We frame the model using the Privacy Vocabulary (Boyle and Greenberg, 2005; Boyle, Neustaedter and Greenberg, $1009)$ to represent the regulation of privacy borders.

The model is structured as three layers of abstraction that connect theoretical concepts of coordination (components) with their corresponding behaviours (mechanisms and 
characterizations), as illustrated in Figure 2. In brief, the first components layer describes collaboration, signalling and grounding in the regulation of interpersonal privacy borders. The mechanisms layer describes the ways in which communicators operate each component to coordinate privacy borders. Finally, the characterizations layer describes the different elements of the mechanisms. These are discussed in turn below.

\subsection{Components}

In the components layer, we classify the different processes by which privacy needs are addressed. These include: the process of collaboration by which the interactors coordinate their communication acts to ensure coherent communication, signalling where privacy intent is communicated in a light way, grounding where efficiency is achieved by creating and using shared representations, and regulation where borders are assessed with respect to individual needs. The first three (collaboration, signalling and grounding) represent the Common Ground elements introduced in PGM. The last component (regulation) maps onto the context in which the model is designed for: the regulation of privacy borders.

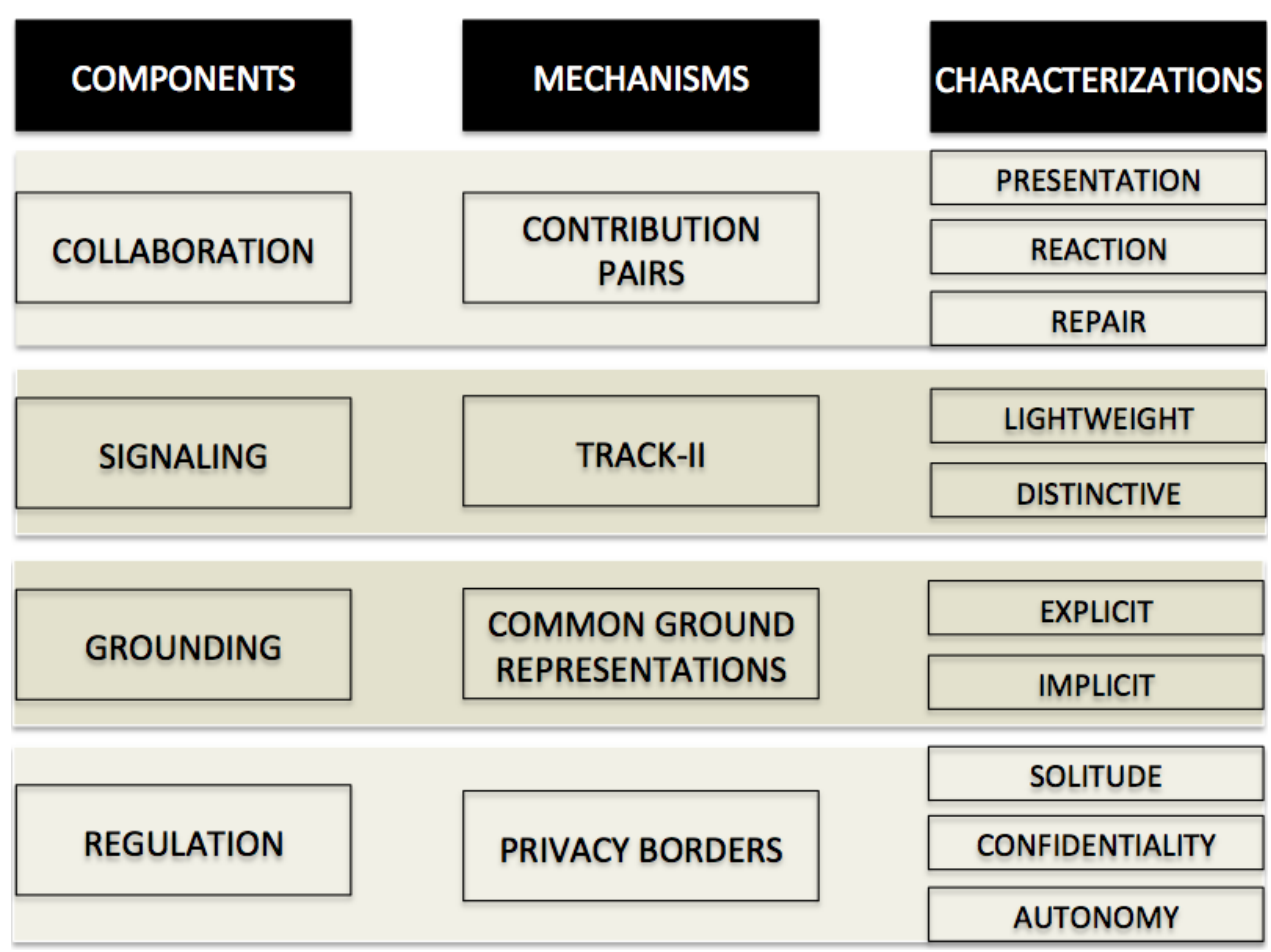

Figure 2: Structure of the Privacy Grounding Model (PGM)

\subsection{Mechanisms}

The second layer of PGM describes the social mechanisms associated with collaboration, signalling, and grounding in the regulation of privacy borders.

The mechanisms to support collaboration are contributions pairs in which both initiators and recipients collaboratively use pairs of presentations and reactions to coordinate privacy intentions as a joint activity. Privacy border coordination consists of at 
least one contribution pair, but it usually involves a sequence of contribution pairs where communicators present and react to privacy meanings and understandings until an agreement has been reached.

The mechanisms to support signalling are track-II signals that communicators use to represent lightweight and distinctive privacy needs when engaging in interaction with others. Example track-II signals in a Instant Messaging system are: updating online status, blurring video, changing presence name in the buddy list, or writing explicitly about one's intentions to interact.

The mechanisms to support grounding are the use and development of common ground representations to establish the necessary shared knowledge. Examples include social rules, shared experiences and shared representations of a situation, and shared knowledge of work; all help the communicators establish an understanding of the situation.

\subsection{Characterizations}

At the third layer, characterizations typify the social interaction space that reflects the choices made by communicators to develop their coordination practices. Such decisions are made depending on the amount of effort communicators wish to invest and how implicit or explicit they want their coordination intentions to be made.

Collaboration choices are characterized by the use of proposals as presentations of intentions to initiate interaction, and acceptances or repairs, as reactions to communicate agreement or to fix a previously produced presentation, respectively. For example, in Instant Messaging communicators could use consecutive instalments (separate by a small time frame) as separate text messages to repair the intention of the message: Hi [enter - 1 minute] I'm back [enter - 1 minute] are you there?. In this case, after each signal, the initiator assessed the (least collaborative) effort to be more distinctive in order to achieve an understanding with the recipient of his intentions to interact with her.

Signalling choices are represented by the four characterizations of track-II signals, classified in lightweight (brief, background, and simultaneous) and distinctive. They describe the effort communicators commit to produce and attend to a coordination signal (in other words how lightweight the signal is), and how implicit or explicit their coordination intentions are made, i.e. how distinctive the signal is.

Grounding choices are characterized by conventions, shared events and external representations. To ensure that grounding is achieved, throughout the communication, communicators assess whether they need to explicitly ground and incur additional effort, or whether they can implicitly ground and therefore minimize the effort needed for grounding.

PGM aims to describe how existing representations in the digital domain can be linked to privacy intentions, via signalling and grounding to develop common ground for privacy coordination (Romero and Markopoulos, 2009).

\subsection{Discussion}

The Privacy Grounding Model emphasizes communicators' needs for lightweight and distinctive collaborative practices to reach the necessary common understanding of their 
privacy intentions. This suggests that technologies should support flexible interactive mechanisms to allow these practices to be played out in a mediated setting.

Signalling and grounding support the collaborative coordination process by providing lightweight mechanisms to establish a common understanding of privacy intentions even in cases when someone is breaching a misunderstood border. For example, if someone initiates communication even after the recipient has blurred her video to represent unavailability, the recipient could explicitly signal her meaning of unavailability intended by the blurred video, which grounds the recipient's unavailability and how it affects the recipient's responsiveness.

A previously described limitation of mediated communication technologies is that representations of collaboration are not always as efficient as in face-to-face settings. For example, collocated communicators can use verbal silence as a lightweight reaction, where its understanding is easily grounded on the basis of other simultaneous signals including body gestures (nodding, leaning forward, etc.) and physical actions (moving away, opening a book, etc.). In mediated communication, silence is rarely presented along with other signals. The lack of simultaneous channels makes it difficult for the recipient to ground silence in a lightweight manner, and for the initiator to deduce the intentions of recipient's silence. To address such situations, PGM elucidates the needs for signalling and grounding that allow lightweight signals such as a silence to be used as acceptable contributions.

The theory of Common Ground describes how people are continuously grounding their intentions to communicate. Similarly, social interactions require different levels of ambiguity to succeed. Aoki and Woodruff (2005) argue that communication systems should allow for ambiguity to support the way individuals manage their self-presentation in their social network, and how they reacted to others. One could think that grounding and ambiguity are the opposite extremes in the spectrum of mechanism to coordinate interaction. However Aoki and Woodruff observed that the preference for providing ambiguous explanations about certain (less collaborative) behaviours could better support grounding than no explanation at all. Begole, Matsakis and Tang (2004) came to the same conclusion, but from a different angle. They developed the Lilsys availability awareness system, which used sensors to detect and display peoples' availability to each other, where their idea was that such representations would make people accountable when initiating or responding to interactions and thus support social norms. Yet they (Begole et. al. 2004) also observed that the information provided was too precise: there was too little room for ambiguity. Compared to common ground, we note that taking ambiguity into account goes beyond the notion of least collaborative effort.

The remainder of this paper presents two case studies that were designed to evaluate whether PGM provides a sufficient and parsimonious description of privacy related behaviours when using particular communication media.

\section{Case Study 1: using the Exodus Instant Messaging System}

We conducted a case study regarding the use of an instant messaging system by a small group of individuals. Our primary research questions were: Is there evidence that communicators coordinate privacy? Can this coordination process be modelled in terms of the Privacy Grounding model? 
The study concerned privacy coordination amongst a group of co-workers using the Exodus instant messaging system. We decided to study privacy coordination over instant messaging for two reasons: communication extends over long periods of time, and communication is restricted to the text medium. In this way, we could analyse the text to see how people coordinate, initiate, close, postpone and decline interactions with others over time. Furthermore, instant messaging systems already provide several mechanisms for declaring one's borders and availability (e.g., buddy-lists, and status indication) and through these we could see how one can acquire, build up and maintain awareness of the availability of others for communication.

\subsection{Description}

The case study concerned the regulation of solitude borders in a community of nine postgraduate students split in two teams, each team working on a different project. Participants were distributed across different rooms of the same building. Members of each team engaged in collaborative activities using various media: Instant Messaging (IM), email and face-to-face communication. The age of participants varied between 25 to 30 years old, including five females and three males. All held a Masters degree in disciplines related to technical and social sciences.

Participants used the Exodus IM client ${ }^{1}$, an Instant Messaging application which provides a buddy list, individual and room chats, and the ability to use pre-defined or customized status indicators. Exodus was installed on all participants' personal computers, with a pre-defined buddy list containing the members of their team. Participants were asked to use Exodus as the primary medium to communicate with other team members for the eight day study period, but they could also use email and face-toface communication if necessary. Participants had the freedom to add more contacts to their buddy list if desired.

Automatic logging was used to collect data of participants' interaction with Exodus and the information exchanged. The logged data was coded using a coding scheme based on the elements of PGM. Each signal was described on four categories: collaboration (presentation or acceptance or repair), signalling (lightweight or distinctive), grounding (explicit or implicit) and regulation of privacy (solitude or confidentiality or autonomy).

Logs were analysed qualitatively to verify whether the coded units (i) could be adequately described as a sequence of contributions pairs of presentations and reactions; (ii) consisting of signalling and grounding mechanisms to establish a shared understanding; and (iii) represented the openings and closings of privacy borders.

\subsection{Results}

Events logged over a period of eight days were classified as user interaction events, system notifications, and other events (see Table 1). Interaction events (33\%) represented interactions where people shared information with other users. These included public or private chat messages as well as setting presence and availability indicators (online status, presence name, presence picture). System notifications (67\%) corresponded to information gathered and displayed by the system. These indicated presence and activity

\footnotetext{
${ }^{1}$ The Exodus system was chosen because it is open source, which allowed us to extend it for the purposes of logging data exchanges. See http://code.google.com/p/exodus/.
} 
of users including automatic status changes, indications of who is typing in a chat room, indications of logging on/off, and indications of joining/leaving public rooms.

Table 1. Pilot results - frequency of events

\begin{tabular}{l|r}
\hline Events & Frequency (unit) \\
\hline Total logged lines & 2734 \\
\hline User Interaction events (33\%) & $\mathbf{8 9 6}$ \\
\hline - Chat & 840 \\
- One-to-one & 588 \\
- Public & 252 \\
- Others & 56 \\
$\quad$ - Manual status & 11 \\
$\quad$ - Others ${ }^{1}$ & 45 \\
\hline System notifications (67\%) & $\mathbf{1 8 3 8}$ \\
\hline - Automatic changes of status & 1027 \\
- Others ${ }^{2}$ & 811 \\
\hline 1 Presence name, picture, and invitation to a public room. \\
${ }^{2}$ Typing, online/offline signals, and joining/leaving rooms.
\end{tabular}

On average, participants sent 72.25 one-to-one messages (std. dev. 55.47). Group-chat participants sent an average of 32.13 messages (std. dev. 42.13). The high standard deviations observed in these interactions reflected the different levels of participation between participants.

\subsection{Analysis}

A total of 265 lines were coded as coordination signals pertaining to initiations of interaction. They were clustered in 103 coordination units. In the logs each unit corresponded to one self-contained episode of privacy coordination consisting of at least on contribution pair (pairs of presentations and reactions/repairs). The coordination signals consisted of 233 interaction signals in the form of text messages ${ }^{2}$, and 32 system's notifications that were (non-text) automatic representations of participants' presence status.

The presence of contribution pairs manifest collaborative coordination: all coordination units consisted of at least one contribution pair to coordinate an initiation (except for 6 initiations that could not be matched to any visible reaction therefore were classified as omissions).

We identified two prevalent collaborative practices by initiators: the use of repair mechanisms to fix one's coordination signals when initiating communication and the use of others' presence status to ground people's availability for communication.

Initiators use repairs to make their signals more distinctive to the recipient when no reaction is received. One example is sending the text message "I'm back" right after their presence status changed to 'online'. Another example is sending a "nudge nudge" text

\footnotetext{
${ }^{2}$ Text messages by definition are not coordination signals as they exchange content. But they might implicitly contribute to the coordination process of a conversation. For this analysis, only the content messages that represented initiations (e.g. John: "Hi, Mary"), reactions to initiations (e.g. Mary: "Hi, John"), and repairs (e.g. John : "Hi, Mary" [1 minute later] "How are you?" [5 minutes later] "busy day, eh?"), were included as track-II signals.
} 
after asking a question to someone, to communicate that a reaction is expected. Occasionally a repair would be used to directly exchange the content of the conversation (e.g. "hi" [1 minute later] "There is a new version of the report that I want you to check"). In such cases content information is transmitted even though the communication has not been yet explicitly established by a reaction of the recipient. PGM describes this "hi" presentation as a coordination signal with the purpose of complying with socially appropriate manners (autonomy control).

Initiators use others' presence status primarily to ground the availability of the recipient: initiators often would not initiate interaction if the status of the recipient showed she/he is unavailable. But they would initiate immediately after an updated representation of the recipient's availability.

Recipients also collaborated in grounding privacy borders. In a few occasions recipients could not react sufficiently fast to an initiation, so they would use short messages such as "one moment", "checking", "mmmh" to avoid a silent delay. In terms of PGM these instances are examples of coordination signals; they reduce the ambiguity as to whether an initiation is known to the recipient or not, creating common ground.

In summary, the practices observed in the loggings showed evidence of a mutual effort by initiators and recipients to facilitate the understanding of their intentions to communicate by grounding meanings to existing representations. In some cases communicators chose to signal their borders explicitly, to coordinate the initiation of a conversation: text signals were used as distinctive coordination signals but not lightweight (non-brief, not in the background, and non simultaneous). In other situations, communicators considered that existing representations in the system were sufficient to signal and ground their availability borders: status and presence messages were used as coordination signals, which were brief, in the background and simultaneous but only distinctive if enough common ground existed to understand the interaction purposes in a particular context.

\subsection{Reflections on the model}

This first field study demonstrated that the components of PGM are, indeed, manifested in mediated communication and are helpful in describing interpersonal privacy border regulation. The study provided evidence that users of at least one communication technology coordinate their interpersonal privacy borders as part of the communicative process they engage in. Collaboration, signalling and grounding represent how communicators signal their intentions and understandings of each others' privacy needs grounding existing representations of privacy borders to achieve the desired state of interaction. The four track-II characterizations (brief, background, simultaneous, and distinctive) helped discriminate and explain the strategies used in grounding privacy borders and in managing the ambiguity in their presentations. Depending on the communicators' needs, existing representations of awareness required or not explicit grounding to be understood as solitude borders or to be considered a sufficient shared basis for indicating privacy intentions.

We do recognize that this study - will providing good evidence - is limited. First, we analysed behaviours related to common ground from text logs from only one week. This relatively short time frame and the low fidelity of text allowed us to gain only limited understanding of existing conventions, shared knowledge and norms within that 
community, which constitute an important part of its existent common ground. Second, participants had to use a system that we supplied, which differed from the one they normally used for their communications. Apart from the adaptation problems that this entails, many participants kept using other messaging applications in parallel to communicate with the rest of their social network; this information was not captured. While our analysis still proved valuable, we decided to study another system already in use by a community, and for a longer period of time .

\section{Case study 2: Community Bar, an extended validation study}

Our second study concerned the interpersonal privacy practices surrounding the use of a desktop awareness called Community Bar. Our community consisted of office workers who were already using it regularly for both work-related and social communication. The first author (referred below as the 'ethnographer') acted as a participant-observer. Detailed logs of interaction with this system were kept for four weeks and were triangulated with ethnographic data gathered by interviews, naturalistic observations, and diary logs.

We observed and analysed the communication behaviours of the community and described their coordination signals using a refined PGM coding scheme. The objective of the analysis was to identify confirming and disconfirming evidence regarding key aspects of the PGM: (a) manifestation of collaborative practices in privacy border regulation, (b) track-II signals use to represent different levels of distinctiveness and lightweight privacy borders, (c) pairing presentation and reaction signals to ground privacy borders representations, and (d) using grounding to regulate solitude, autonomy, and confidentiality borders.

\subsection{Study design}

The community under scrutiny had already been using the Community Bar (CB) system for two years. Its design was intended to support awareness and casual interaction in small communities (McEwan and Greenberg, 2005; McEwan et al., 2006). The group included experienced and novice CB users. Three of the fifteen participants of this group had worked on the development of CB, while two others had

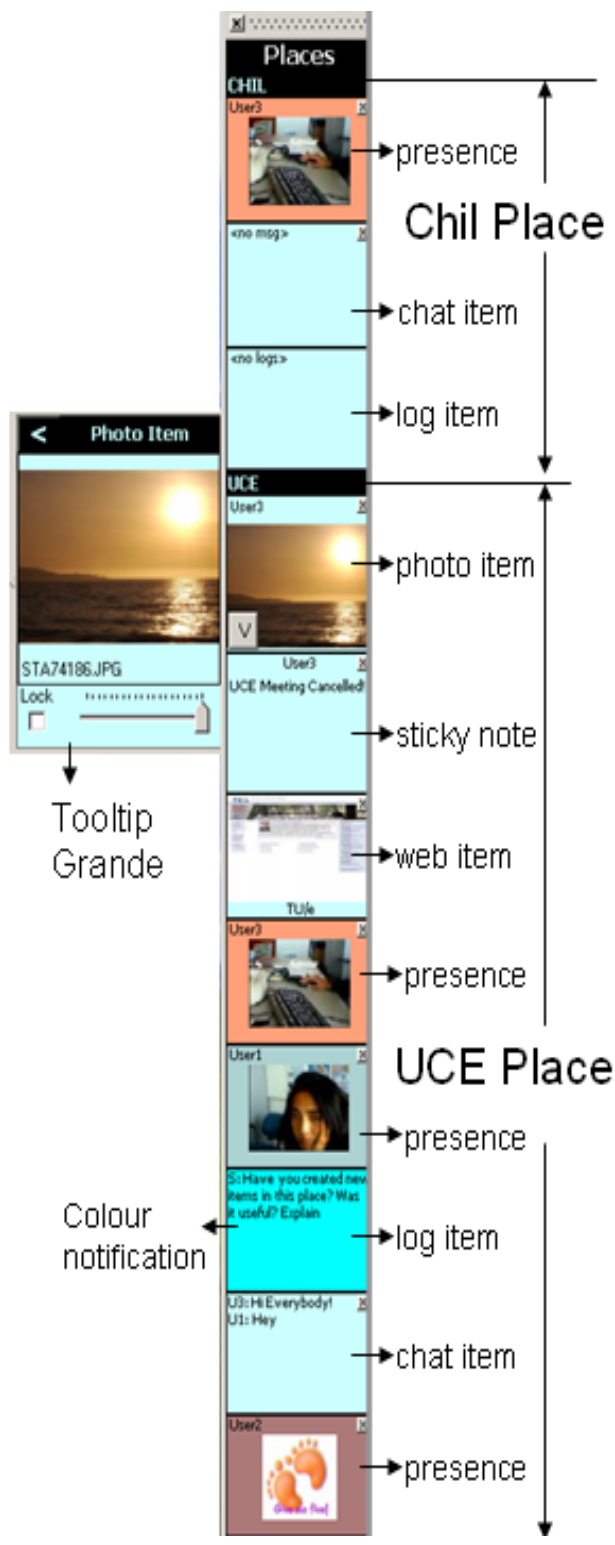

Figure 3: Community Bar - sidebar 
been involved in the design of the Notification Collage (Greenberg and Rounding, 2001), a predecessor to the $\mathrm{CB}$ system studied here. The other 10 had no or minimal involvement with it.

The fifteen participants formed a small cohesive academic group: eleven of them worked at the same research laboratory, three were former graduate students now working for other companies or institutions (two of them in different cities) and one member was an external researcher contacting other members of the community only occasionally. The eleven collocated members consisted of one Professor and ten Master and $\mathrm{PhD}$ students, out of which five were under the Professor's direct supervision. As mentioned, all members had been using the system prior to the study, ten of them frequently and four intermittently. The ethnographer joined as a temporary member of the community for a period of four months; she had used the system for one month prior to the actual logging of system interactions reported below.

The community's physical work environment consisted of an open area for students, and few offices around it for the professors and meeting rooms. Four members telecommuted twice a week. Master and $\mathrm{PhD}$ students would often stay until late in the evening, or come during weekend to work, or do social activities together (e.g. sports or play computer games).

At the time of this experiment, members of the community used $\mathrm{CB}$ on a voluntary basis. Its main benefits were its high fidelity awareness of those who were online (via regularly updated snapshot video and/or status indicators) and its video and textual communication capabilities. People usually used it to engage in brief work or socially related interaction. The telecommuters, like the Professor, had special interest in using $\mathrm{CB}$ as it provided the possibility to easily reach other colleagues and students when working from home. Most participants used $\mathrm{CB}$ as the main channel to connect with colleagues, but not exclusively. The public nature of the system (everything is broadcasted to everyone) and its restricted audience (limited only to the particular 15 members in the research community) led most participants to use other communication applications as well. Email and Instant Messaging (IM) systems allowed CB users to engage in more private (one-to-one) conversation as well as to reach a larger number of people.

A webcam-supplied regularly updated image provided others with one's video presence. However, its use was recommended but not obligatory. Most participants had web cameras installed at their desks, and the ones who did not could optionally request one for the study period. Only four participants did not use a webcam during the study: two because of technical problems or company policies; and two because of personal choice. Participants were asked to use $\mathrm{CB}$ as usual, where they could also use other communication media if desired.

The role of the ethnographer during the four months stay in the lab included the following tasks: individual interviews to eight group members with the purpose to learn about the community, its members, and their relation with $\mathrm{CB}$ (first month); the implementation and execution of the study, limiting the ethnographer's interaction with the community outside $\mathrm{CB}$ (second and third months); individual interviews to eleven participants to discuss their experience with $\mathrm{CB}$ during the study period (fourth month).

An adapted version of Community Bar was installed in participants' desktop and laptop computers. Figure 3 illustrates some of its features. The Community sidebar is an 
always-visible bar positioned at the side of the screen (it remained visible even if other applications were set to maximized view). The sidebar provides a shared space where users can post and see 'media items' - interactive media that could be posted by community members - as small tiles. Media items could take the form of video, text notes, web pages, availability status, digital photos, etc. The design intent of the sidebar's position on the screen and its media item content was to provide information about the whereabouts and activities of others at a person's periphery of attention. When a person passes the mouse over a the tile view of the media item, a larger 'tooltip grande' appears (Figure 3, left side) containing more information and/or interactive controls. The user could also double click that tooltip grande to raise a full-sized window running the highest fidelity and most interactive version of the media item.

Visual notifications were provided to inform users about new items and updates of existing items. A notification turns the background colour of the tile view of the new or updated item brighter until the new information is acknowledged by the viewer (by moving the mouse over the tile).

The media items available at the time of the study were:

- Chat items, representing a textual space for public conversations;

- Presence items, representing presence of people (optionally as video snapshots) connected to the system;

- Sticky notes, used as public static text;

- Web items, to publish web URLs;

- Photo items, to share pictures.

The adapted version also had a buzz button in the presence item, to claim the attention of others. Clicking the buzz button of the presence item of the person to buzz (the target), generated a visual notification in the target's sidebar; the presence item of the buzzer flashed for a few seconds or until the target user moved the mouse over it. This option was implemented in response to the wishes of $\mathrm{CB}$ users, where they wanted an explicit mechanism to coordinate the initiation of an interaction. In addition, a log item was implemented as a data logger, which prompts question to encourage participants report on their experience using the system.

\subsubsection{Data Collection}

We used four methods to gather qualitative and quantitative data regarding users' interaction practices, their understanding of privacy representations in the system, and their reflection on privacy related issues.

First, a data logger was implemented to collect data on the use of the system. It registered participants' interactions with the system by collecting both context information (time, place, subject, target) and content information depending on the media item (text, URL, image file, etc.)

Second, diary logs captured in-situ reflections by participants about their experiences with the system. The diary was implemented as a media item in CB (see 'log item' in Figure 3), autonomously prompting generic and event-related questions to encourage participants' contribution. It randomly selected a general question every hour and sent it to all users. In addition, event-related questions were prompted only to the participants involved in the specific event soon after it took place. 
Third, direct naturalistic observation helped the researcher acquire insights regarding the community, its structure, and the type of relationships between its members and the embedding of CB usage in these.

Finally, open in depth interviews were conducted twice. At the beginning of the study, we interviewed eight members of the community, some actively using CB and others not. At the end of the study, we individually debriefed eleven participants via semi-structured interviews, focusing on their interpersonal interactions and privacy concerns when using CB. Participants were asked to comment on: perceived benefits from CB; the ease and effectiveness of assessing others' reachability in $\mathrm{CB}$; the use of social rules to assess acceptable behaviours; and whether collaboration was considered necessary to achieve a satisfactory use of the system. All interviews were audio-recorded, transcribed and analysed qualitatively.

\subsubsection{Analysis Procedure}

We used standard qualitative analysis methods to identify and describe instances of initiation of interaction and reaction to initiations. The instances of interaction (from now on called 'signals') were coded using a redefined version of the coding scheme used in the first case study.

The coding procedure was as follows. First, we identified contribution pairs by describing every signal as a presentation (initiation), reaction or repair, and coupled with another signal to form a contribution pair. Second we describe the signals using the four track-II characterizations indicating whether lightweight (brief, background, simultaneous) and distinctiveness were present or not in the signal, using 'yes' (+), 'no' () or 'to some extent' (+/-). For the grounding component, all signals were characterized as whether they explicitly ground (E) or implicitly ground (I).

The data logger captured a total of thirteen types of contribution depending on the media items available. From the presence item the logs collected updates of video snapshots, availability status (manual and automatically set), picture, buzz notifications and text message. Data collection from the chat item included the sender, content and time of new messages. From the web, sticky notes and photo items, collected data included the sender, content and time of urls, notes, and photos respectively. Finally the logs also captured the time when users logged on and off the system.

We drew a distinction between media items that are primarily suited for content exchange (track I) and those suited for coordinating the communication (track II). (But recall that the first case study showed that participants signalled coordination contributions (track-II) using content channels (e.g., a text message "I'm back"), most likely to overcome the lack of appropriate track-II channels for coordination purposes). We characterized chat, photo item, sticky notes and web items as content channels and presence messages, status, video and picture updates as coordination channels.

The analysis procedure included two coders and used the cross-checking technique (Miles and Huberman, 1994) to increase the reliability of the analysis. We calculated the intercoder reliability using Miles and Huberman formula (1994, pp.64), which provides a ratio of the total number of agreements to the total number of codes (agreements plus disagreements). The coded data was then triangulated with the information gathered from diary logs and interviews finding relations between the coded patterns and participants' attitudes and reflections on their behaviours in CB. 


\subsection{Results}

The average of users logged per day during the first three observation weeks was stable (8.1, 7.4, and 6.6 respectively), but it dropped down to 3.6 in the fourth week due to holidays. The peak attendance was 11 users logged on at the same time in the second and third weeks. The peak attendance was observed during weekdays between 09.00 and 18.00. In the afternoon intervals (from 12.00 to 15.00) between 6 to 10 people were online. Several people logged on the system during evening intervals (from 18.00 till midnight).

Table 2 presents the frequency of the coordination signals used by the participants ${ }^{3}$. Coordination signals were classified as either system notification or user interaction. System notification signals $(96 \%)$ represented information that was broadcasted automatically by the system, like video, automatic change of status, and online/offline indicators. User interaction signals $(4 \%)$ represented information generated by explicit user's actions in the system. Most of the user interaction signals occurred in the content channels $(94 \%)$, where chat message was the prominent signal observed $(98 \%)$. The remaining $6 \%$ of user interaction signals corresponded to signals in the coordination channels with presence message (49\%) and buzz (30\%) being the most frequently used.

Table 2. Frequency of signals

\begin{tabular}{l|r}
\hline Signals & Frequency (unit) \\
\hline System notifications & $\mathbf{2 4 2 6 0}$ \\
\hline Video & 21975 \\
Automatic Status & 1922 \\
Online/Offline & 363 \\
\hline User Interactions & $\mathbf{1 0 1 7}$ \\
\hline In content channels & $\underline{960}$ \\
\hline Chat & 945 \\
Photo, sticky notes, web & 15 \\
In coordination channels & 57 \\
Presence message & 28 \\
Buzz & 17 \\
Manual Status & 8 \\
Presence picture & 4 \\
\hline Total coding lines & $\mathbf{2 5 2 7 7}$ \\
\hline
\end{tabular}

\subsection{Analysis}

We identified 501 contribution signals related to privacy coordination, which corresponded to initiations, reactions, omissions, delays, and presence signals that trigger initiation. Delays included reactions to an initiation that was sent ten minutes before or longer. The threshold of ten minutes was set to reflect the intermittent nature of chat conversations where reactions of about up to ten minutes are quite usual. Reliability rates calculated for each coding category (collaboration, signalling and grounding) were higher

${ }^{3}$ Diary entries were not considered signals for the coding analysis, therefore they are not present in Table 4 . The response rate was $15 \%$, with a highest number of entries in the first: 29.3 average per day. 
than $80 \%$, which is very satisfactory; no disagreements remained after discussion (a more detailed report can be found in (Romero, 2008)).

\subsubsection{Collaboration}

Participants' contributions to initiate communication varied widely. The daily average of initiations per participant was nine. Three participants never initiated any conversation (P3, P4, and P6). The interviews and diaries indicated that participants developed different collaboration strategies depending on the nature of their membership in the community. On the one hand, the 'closer' a person was to the core community ${ }^{4}$, the more common ground already existed, and therefore the less distinctive but more lightweight signals are used for coordinating initiations. On the other hand, explicit collaboration using more distinctive signals (and that made interaction somewhat more heavyweight) was recognized by the core group as a social conduct to maximize awareness and interaction benefits.

"What I would usually do is a very quick thing like saying I'm on the phone ... usually I kind of say I hear you but not now ... and sometimes I say I can't talk now ... I usually kind of point to the phone in the video so it gets updated" (Interview Participant I3)

Even in the situations in which individual behaviours conflicted with the general social practices, collaboration was considered important. For example, a participant's decision to not use video was mostly compensated by this person expending extra individual effort to maintain a desirable level of connection with the community. Otherwise such participants felt being treated as second-rate participants and experienced a decrease in their privileges as full members.

"Because not having a webcam does require more effort [for me] to project [my] status" (Interview Participant 10).

The use of delayed reactions was another example of conflicting expectations between the community and the individuals. Participants reported that delays (18\%) and omissions $(17 \%)$ were acceptable coordination mechanisms; in addition the logs showed that most delays as well as omissions of initiations were not explicitly confronted with messages such as "why are you not responding. Contrastingly participants also reported that such mechanisms were less optimal contributions as they left them wondering why is he/she not responding.

In summary, the need to acknowledge non-collaborative mechanisms confirms that privacy border regulation was not just about allowing or disallowing communication, but also about repairing 'damage' after a certain communicative attempt was neglected. The need to repair could be motivated by politeness, but in most cases the purpose to repair related to the need of balancing a situation where less collaborative behaviours were used (e.g. not using video or not responding a message).

\subsubsection{Signalling}

Coordination signals were described as lightweight depending on the brief, background, and simultaneous track-II characterizations, which reflected the effort needed to produce a signal: we consider an automatic status change lighter than a text message. The

4 The distance to the community was defined considering their work/social and physical distance from the core group. A person could be physically further away but socially close. 
distinctiveness characterization of the signal reflected the effort needed to understand whether a signal was meant as a coordination signal or not.

Following the classification of the 501 coordination signals as being produced in content or coordination channels, we identified four different characterizations regarding lightweight (brief (br), background (ba), simultaneous (si)) and distinctiveness (di) (see Table 3).

\begin{tabular}{|c|c|c|c|c|c|c|c|}
\hline \multicolumn{4}{|c|}{ Coordination Channel (58\%) } & \multicolumn{4}{|c|}{ Content Channel (52\%) } \\
\hline $\mathrm{Br}$ & $\mathrm{Ba}$ & $\mathrm{Si}$ & Di & $\mathrm{Br}$ & $\mathrm{Ba}$ & Si & $\mathrm{Di}$ \\
\hline+ & + & + & - & + & - & + & - \\
\hline \multicolumn{4}{|c|}{$\begin{array}{l}\text { System generated presence } \\
\text { representations that are used to } \\
\text { coordinate the initiation or end of an } \\
\text { interaction }(30 \%)\end{array}$} & \multicolumn{4}{|c|}{$\begin{array}{l}\text { Text signals that initiates interaction } \\
\text { by directly contributing to the } \\
\text { conversation }(13 \%)\end{array}$} \\
\hline $\mathrm{Br}$ & $\mathrm{Ba}$ & Si & $\mathrm{Di}$ & $\mathrm{Br}$ & $\mathrm{Ba}$ & $\mathrm{Si}$ & $\mathrm{Di}$ \\
\hline+ & + & - & - & - & - & - & + \\
\hline \multicolumn{4}{|c|}{$\begin{array}{l}\text { User generated presence } \\
\text { representations that are used to } \\
\text { coordinate the initiation or end of an } \\
\text { interaction }(28 \%)\end{array}$} & \multicolumn{4}{|c|}{$\begin{array}{l}\text { Text signals that seek establishing a } \\
\text { share understanding of intentions to } \\
\text { interact }(29 \%)\end{array}$} \\
\hline
\end{tabular}

Table 3: Coordination signals characterizations based on channel

In coordination channels participants communicated their intentions to interact based on two types of presence information: system generated signals (video broadcast and automatic changes of online status) and user generated signals based on self-presentation status (presence message, online status, and picture). The former were considered lightweight signals (brief, in the background, and simultaneous), while the latter were less lightweight since they could not be generated simultaneously to content signals (changing one's status required participants to select a new status from a checkbox or adding or removing text). Although participants created presence signals to communicate intentions of interaction (e.g "I'm away"; "I'm in a meeting") both system and user generated signals were characterized as not distinctive. In the analysis, the effectiveness of such signals was related to the existing common ground among the parties involved. In cases when common ground was not sufficient, participants engaged in extra effort to understand the coordination purpose of a signal. For example, in some cases a blurred video required explicit coordination to understand one's intentions of interaction. Yet in other cases that blurred video was sufficient for one party to understand that the other party was using it to communicate conversation unavailability.

In content channels, participants created two types of text messages to communicate their intentions to interact. If no explicit coordination was considered necessary, communicators initiated their interaction directly and thus contributied to the content of the conversation. If explicit coordination was needed, participants typically exchanged text messages before content was communicated to first ensure a shared understanding of their availability. The former was characterized as brief and simultaneous to the content signals but not in the background, as the initiator pushed the initiation without using track-II signals (or ignoring existing ones). Similarly to what was observed in coordination channels, these types of signals were only distinctive when sufficient common ground existed. The latter signals were described as distinctive but non- 
lightweight contributions, used by participants primarily to ground existing coordination signals. The following contribution shows an example: P9 writes "are you at home? Is that why shields [of video camera] are up?"; P1 reacts "yah at home but actually forgot about the shield"; [P1 opens the shield]; P9 reacts "there you are! Still a tad blurry"; P1 reacts "yah, I adjusted the focus of the camera" Similar text signals to acknowledge a delay or one's presence status were in this category.

Distinctiveness seemed to be necessary to guarantee the effectiveness of coordination signals, as the meaning of signals varied over time and between participants. For example, in some occasions an 'away' status meant "I am not in my office" therefore a delay was most likely to be expected, while in other situations it meant "I am in my office but away from my screen" so a sooner answer could be expected. We observed that distinctive track-II signals were non-lightweight (less brief, less in the background, and less simultaneous) but they were necessary when less distinctive signals failed in grounding privacy intentions. The signals described as $(--++)$ represented this behaviour. In content channels, text messages intended to coordinate availability were considered not lightweight but highly distinctive in communicating their intentions to interact.

\subsubsection{Grounding}

We identified two types of grounding, where the type depended on the effort spent in grounding. Participants used existing signals to implicitly (without an explicit action) ground theirs or others' intentions for interaction. The success of this type of grounding depended on the existing common ground of the parties involved. A design implication to support this type of grounding is to provide a good variety of lightweight signals that people can use to ground availability.

However, despite the fact that $\mathrm{CB}$ offers a wide variety of signals to support implicit grounding, participants also spent effort to explicitly ground their intentions to interact. Using PGM we described explicit grounding as how participants used non-lightweight distinctive signals $(---+)$ to develop common ground representations. In CB they represented the use of clicks (to change online status or buzz others) and texts (to explicitly communicate intentions to interact).

Two types of explicit grounding were observed with the used of $(---+)$ signals: to ground existing signals or to create new signals as common ground representations. Creating new representations was mostly described as texts messages that did not refer to an existing signal (or ignored the presence of other signals), e.g. "I want to ask you a question" (even if the recipient showed to be unavailable). The need of creating new signals was limited to urgent or very specific type of interactions.

Grounding existing signals included making sure that the signal was a shared signal to everyone (e.g. "did you ping me?", "I'm back online"), or that everyone reached a shared understanding of its meaning (e.g. "why are you [is your video] blurred?"). The need of grounding existing signals shows that participants need to ground their meaning as privacy borders representations, as the distinctiveness of signals is highly dynamic and subjective. Without grounding, the meaning of existing signals as privacy border representations was likely to be misunderstood or unnoticed when coordinating a particular instance of interaction. 
Delays and omissions were described as lightweight and implicit grounding, considered as acceptable mechanisms as in most cases no visible repairs were observed afterwards. Yet conflicting evidence was found in the interviews: participants reported that 'waiting' and 'delaying' was perceived as a frustrating experience, indicating the need to ground such signals. Participants explained that, when expressing their unavailability by delaying a reaction it was interpreted as ignoring or missing the initiation, with no possibility to establish an understanding of the delay without using text messages. Similarly, when confronted with a delay they did not have enough information to decide whether to wait or to insist to get the desired attention.

\subsubsection{Regulation}

Our analysis also describes how participants engaged in coordination activities to regulate their needs for interaction (solitude), information about themselves (confidentiality), and their identity within the community (autonomy).

The most common example of confidentiality control was participants' use of presence signals (video and presence messages) to adapt their self-disclosure, i.e., where they increased or decreased the amount of information about themselves made available via the system to others. For example, participants used non-video or blurred video settings to coordinate confidentiality by reducing their representation to others.

As we have described earlier, solitude borders were coordinated with different levels of distinctiveness. For example, in many occasions participants added text under their presence representations (whether a picture or video) which gave the opportunity for others to make an educated guess of when to interact with them. Similarly, the way participants use their video could give away their intentions for interaction. A more distinctive way of coordinating solitude was the use of the buzz function. Often buzz was used to repair a previous initiation that had been ignored or omitted, emphasizing the need to get someone else's attention.

The flexibility to not use video, or the use of delays and omissions provided evidence that autonomy control was necessary by their members. This is so even though some members characterized this behaviour (to some extent) as 'non-collaborative' practices. As previously reported, in most cases autonomy control was accompanied by mechanism (explicit grounding) to compensate such 'insufficient' social practices within the community.

\subsection{Conclusions}

The field study has shown that PGM components can provide a detailed account of the process by which (existing) representations of privacy borders are grounded. Signalling privacy borders ensured that its representation became a shared knowledge, so it could be recognized by everyone as a privacy border. Grounding a privacy border ensured that its representation was mutually understood, so that everyone could use it as common ground in the coordination process.

In particular, we demonstrated how coordination channels provide relevant information to represent privacy borders. However, in most cases communicators needed mechanisms to explicitly ground the privacy implications of such representations. Therefore, a tradeoff exists between the use of lightweight coordination channels and the presentation of distinctive coordination signals. Our first conclusion indicates the need to design 
lightweight and interactive coordination channels thus providing opportunities for communicators to reach a shared understanding of their coordination signals dynamically and with low effort.

The collaboration component of PGM characterized coordination signals as sequences of presentations and reactions that varied in their level of distinctiveness. If the needs for coordination required special actions, a collaborative effort was represented by a sequence of distinctive signals to develop the necessary common ground representations. If there was no need for such distinctiveness, the required effort was minimized by a sequence of less distinctive contributions used as existing common ground. For example, a user might rely on the automatic 'away' or 'idle' representation of his online status to coordinate his desire for solitude, assuming that others share its meaning. If someone was trying to reach him anyway, he might then use more distinctive signals to explicitly indicate his unavailability to others.

It is important to note that regardless the level of distinctiveness, contributions were considered in this analysis as collaborative practices as soon as both communicators reached the same understanding. Overall, participants considered the use of less distinctive contributions as less collaborative if a minimum shared understanding had not yet been established. Similarly, they perceived the use of distinctive contributions as less collaborative when the existing common ground was perceived sufficient but failed to be properly shared. For example, Anne is not responding to Pete's messages, though she understands Pete's intentions but she is not able to react immediately. The system fails to provide Anne with lightweight/interactive mechanism to share her understanding and intentions to Pete. In reaction, Pete added more distinctive messages to convey more explicitly his intentions to her. Anne considered such messages unnecessary (less collaborative) and possibly interruptive.

\section{Design Case}

Implementations of signalling and grounding mechanisms in Community Bar (Romero and Markopoulos, 2008; Romero, Boer, and Markopoulos, 2008) have provided first insights of how lightweight and interactive techniques help individuals develop common ground over their interactions needs. In related work, the evaluation presented by Romero and Markopoulos (2008), analyzed the use of a 'one-click' grounding mechanism which allows users of Community Bar to click on an existing chat message, making this action a shared representation to others (see Figure 4). 'One-click' was designed to minimize the collaborative effort by providing a light technique to react to existing chat representations. It supports intentional signalling of privacy intentions as 'the one-click' reaction is linked to the clicked message through a visual representation. It maximizes the effectiveness of the mechanism by ensuring that the visualization of the grounded message indicates the name (initials) of the grounder that has clicked on the message. 


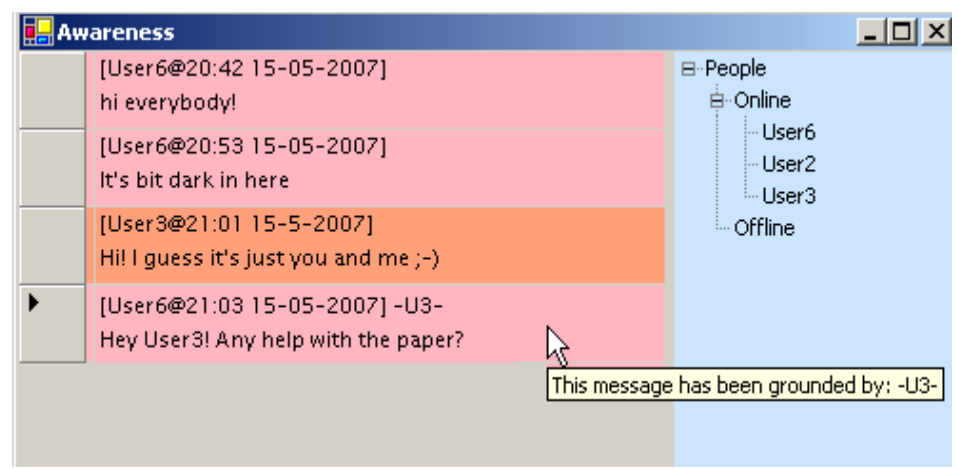

Figure 4: 'One-click' mechanism: User 3 (U3) has clicked a message of User 6

An interesting collaborative behaviour that emerged from the use of the one-click was to ground passive participation: participants could acknowledge with one-click that they were aware about a particular conversation but without having to actively participate in it.

Other mechanisms developed are the 'drag\&drop pallet', where predefined messages can be dragged and dropped under existing messages, allowing a more distinctive grounding than the 'one-click'. Finally, we are also exploring tangible mechanisms to make more distinctive the grounding interactions, moving the grounding outside the digital content channel. The 'Cylinder' was designed to provide lights and movements for signalling and pressing and turning for grounding interactions (see Figure 5). When someone gets the attention of someone else via CommunityBar, the cylinder goes up indicating to the recipient who is asking for attention (coloured lights). By pressing the cylinder the recipient grounds their availability: immediate press means not available, turning clock-wise means available after a certain period, no press means no grounding.

(1)

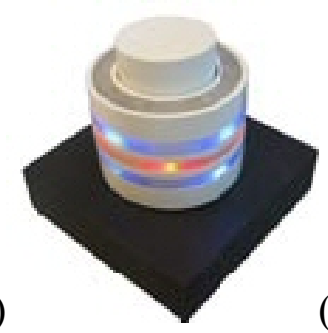

(2)

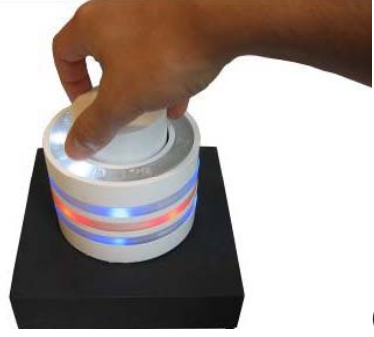

(3)

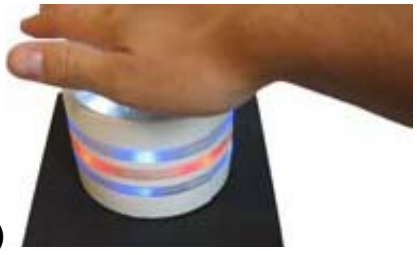

Figure 5: The 'Cylinder' tangible grounding mechanism: (1) signalling one's attention; (2) setting available timeframe (optional) ; (3) grounding action

\section{Conclusions and Future work}

Interpersonal privacy management has become a growing concern in the design of technologies supporting mediated communication. Users are often confronted with difficulties regarding interruptions and managing their availability for communication. A common approach to address these issues has been primarily concerned with predicting users' availability and automating the interaction control based on their privacy preferences. From a social interaction perspective such solutions appear ill-fated as privacy should be treated as a dynamic and collaborative regulation process in which communicators open and close their interaction borders to represent and understand their mutual privacy needs.

Following the above conception of privacy, we have investigated, proposed and validated the Privacy Grounding Model as a way to describe and analyse the process by 
which communicators cooperate to convey, adjust and mutually agree on a desired level for solitude or for socializing. The model identifies signalling and grounding mechanisms as collaborative and dynamic practices in the coordination of interpersonal privacy borders. Further, we have shown that in order to support the signalling and grounding of privacy borders lightweight interactive mechanisms are needed. Such mechanisms such mechanisms should not compromise the ambiguity in the presentation of privacy interaction borders to maintain people's autonomy.

We hope that this research contributes to a better understanding of the dynamic and collaborative process of interpersonal privacy coordination and that it can inform design of future mechanisms supporting that process, like the implementation of the 'one-click' mechanism.

Our analysis is suggestive of manual coordination of privacy borders. Further research could explore the role of automation in supporting this process. It is clear that explicit grounding in systems supporting mediated communication requires exploring a range of disambiguation signals when automatically constructed status updates are used for privacy border regulation. Belloti et al. (1993) offer a list of requirements that could be used to check whether grounding mechanisms support things such as: appropriate feedback timing (as when control is most likely to be required and effective), perceptibility, unobtrusiveness, flexibility, meaningfulness, and learnability (solutions should be sensible to existing psychological and social mechanisms). Palen et al.,(2003) define genres of disclosure to describe the expectation and response around sociotechnology arrangements that are involved in everyday privacy management. Genres of disclosure could be used to check whether grounding representations address tensions in disclosing public and private information, identity of the self and other and temporality (past and future).

In future analysis, more attention could be dedicated to understand the effect of grounding on ambiguity. The main question is how could the process of grounding not hinder ambiguity when desired. If we look at the main benefits of grounding, we observed that communicators collaborate to minimize uncertainty. Our findings showed no evidence of participants questioning the validity of others' privacy borders after an understanding was reached. In line with Aoki and Woodruff's (2005) results, this gives insights on the possibilities to acquire grounding by helping to minimize uncertainty without compromising undesired interaction. Therefore, explicit grounding could "preserve" ambiguity by supporting the possibility to associate fake or multiple meanings to contributions of privacy borders. Supporting flexible and lightweight grounding mechanisms decreases uncertainty and increase collaboration and interactive ways to achieve efficient and effective coordination of interpersonal privacy.

\section{Notes}

Background: the reported research based on Romero's doctoral thesis done at TU Eindhoven.

Acknowledgements: we thank our experimental participants for their participation in our studies.

Support: The GroupLab research group (Computer Science Department, University of Calgary) and the User Centered Design research group (Industrial Design Department, 
Eindhoven Univerisity of Technology) for providing all the facilities needed to conduct this research

Authors' present addresses: Natalia A. Romero, Industrial Design Engineering Faculty, Delft University of Technology, Landbergstraat 15, 2628 CE Delft. E-mail: n.a.romero@tudelft.nl. Panos Markopoulos, Industrial Design Department, Eindhoven University of Technology, Den Dolech 2, 5600MB. E-mail: p.markopoulos@tue.nl. Saul Greenberg, Department of Computer Science, University of Calgary, Calgary, Alberta, Canada T2N 1N4. E-mail: saul.greenberg@ucalgary.ca.

\section{References}

Altman, I. (1975). The Environment and Social Behaviour - Privacy, personal space, territory, crowding. Monterey (Ca), Wadsworth

Aoki, P. M. and Woodruff, A. (2005). Making space for stories: ambiguity in the design of personal communication systems. In Proceedings of the SIGCHI Conference on Human Factors in Computing Systems, CHI '05. ACM, pp. 181 - 190.

Begole, J. B., Matsakis, N.E, and Tang, J. (2004). Lilsys: Sensing Unavailability. In Proceedings of the 2004 ACM Conference on Computer Supported Cooperative Work, CSCW'04, pp. $511-514$.

Bellotti, V. and Sellen, A. (1993). Design for privacy in ubiquitous computing environments. In Proceedings of the third conference on European Conference on Computer-supported cooperative work, ESCW' 93, pp. 77 - 92.

Bly, S., Harrison, S., and Irwin, S. (1993). Media Spaces: Bringing People Together in a Video, Audio and Computing Environment. In Communications of the ACM, vol. 36 (1), pp. $28-46$.

Boyle, M., and Greenberg, S. (2005). The Language of Privacy: Learning from Video Media Space Analysis and Design. In ACM Transactions on Computer-Human Interaction (TOCHI), vol. 12 (2), pp. $328-370$.

Boyle, M., Neustaedter, C. and Greenberg, S. (2009) Privacy Factors in Video-based Media Spaces. In Media Space: 20+ Years of Mediated Life. (S. Harrision, Ed.), Springer, pages 97-122.

Clark, H. (1996). Using language. New York, Cambridge University Press

Dourish, P. and Bly, S. (1992). Portholes: supporting awareness in a distributed work group. In Proceedings of the ACM Conference on Human Factors in Computing Systems, CHI'92, pp. $541-547$.

Dourish, P. (1993). Culture and Control in a Media Space. In Proc European Conference on Computer-Supported Cooperative Work. ECSCW'93, pp. 125 - 137.

Erickson, T., Smith, D. N., Kellogg, W. A., Laff, M., Richards, J. T., and Bradner, E. (1999). Socially translucent systems: social proxies, persistent conversation, and the design of "babble". In Proceedings of the SIGCHI Conference. CHI '99, pp. 72-79.

Garde - Perik, E.M. van de, Markopoulos, P., de Ruyter, B, Eggen, B., IJsselsteijn, W.A. (2008). Investigating privacy attitudes and behavior in relation to personalization. Social Science Computer Review, 26(1), 20-43. 
Greenberg, S., and Rounding, M. (2001). The notification collage: Posting information to public and personal displays. In Proceedings of the SIGCHI Conference on Human Factors in Computing Systems. CHI '01, pp. 514 - 521.

Harper, R. H., Lamming, M. G., and Newman, W. M. (1992). Locating systems at work: implications for the development of active badge applications. Interact. Comput. 4, 3 (Dec. 1992), pp. 343-363.

Lederer, S., Hong, J., Dey, A., and Landay, J. (2004). Personal privacy through understanding and action: five pitfalls for designers. In Journal of Personal and Ubiquitous Computing, vol. 8 (6), Springer, London, pp. 440 - 454.

Mackay, W. (1999). Triggers and barriers to customizing software. In Proceedings of the Conference on Human Factors in Computing Systems, CHI '99, pp. 153 - 160.

Markopoulos, P., Romero, N., van Baren, J., IJsselsteijn, W., de Ruyter, B., and Farschian, B. (2004). Keeping in touch with the family: Home and away with the ASTRA awareness system. In Proceedings CHI 2004 extended abstracts on human factors in computing systems, pp. $1351-1354$.

McEwan, G. and Greenberg, S. (2005). Supporting social worlds with the community bar. In Proceedings of ACM. Group '05, pp. 21 - 30.

McEwan, G., Greenberg, S., Rounding, M., and Boyle, M. (2006). Groupware plug-ins: A case study of extending collaboration functionality through media items. In Proceedings CollabTech. IPSJ SIG 2006, pp. 42 - 47.

McFarlane, D. C. (2002). Comparison of Four Primary Methods for Coordinating the Interruption of People in Human-Computer Interaction. In Human Computer Interaction, vol. 17 (1), pp. 63 - 139

Metaxas, G., Metin, B., Jutta Schneider, J., Markopoulos, P., and de Ruyter, B. 2007. Daily Activities Diarist: Supporting Aging in Place with Semantically Enriched Narratives. In Proceedings of INTERACT 07 Human Computer Interaction, pp. 390 -403 .

Miles, M. and Huberman, M.(1994). Qualitative data analysis: an expanded sourcebook. Sage Publications.

Miller, T. and Stasko, J. (2001). The InfoCanvas: information conveyance through personalized, expressive art. In CHI '01 Extended Abstracts. CHI '01 pp. 305-306.

Monk, A. (2003). Common ground in electronically mediated communication: Clark's theory of language use, in J.M. Carroll (Ed.), Toward a multidisciplinary science of HCI. 265--290. MIT Press.

Nardi, B., Whittaker, S., and Bradner, E. (2000). Interaction and outeraction: instant messaging in action. In Proceedings of the 2000 ACM Conference on Computer Supported Cooperative Work. CSCW '00. ACM, pp. 79 - 88.

Palen, L. (1999). Social, individual and technological issues for groupware calendar systems. In Proceedings of the SIGCHI Conference on Human Factors in Computing Systems. CHI '99. ACM, pp. $17-24$. 
Palen, L., and Dourish, P. (2003). Unpacking "privacy" for a networked world. In Proceedings of the SIGCHI conference of Human factors in computing systems, pp. $129-136$.

Patil, S., and Kobsa, A. (2004). Instant messaging and privacy. In Proceedings of Human Computer Interaction, HCI 2004.

Petronio, S. (2002). Boundaries of privacy: dialectics of disclosure. Albany, State of University of New York Press.

Reichenbach, M., Damker, H., Federrath, H., and Rannenberg, K. (1997). Individual management of personal reachability in mobile communication. In Proceedings of the IFIP TC11 SEC'97 on Information security in research and business, pp. $164-$ 174.

Romero, N., and Markopoulos, P. (2005). Common Ground to Analyse Privacy Coordination in Awareness Systems. In Human-Computer Interaction - INTERACT 2005, pp. $1006-1009$.

Romero, N., Markopoulos, P., van Baren, J., de Ruyter, B., Ijsselsteijn, W. and Farshchian, B. (2007a). Connecting the family with awareness systems. In Personal Ubiquitous Computing, 11 (4), pp. 299 - 312.

Romero, N., Matysiak, A., Kaptein, M., and Markopoulos, P. (2007b). Behaviours and preferences when coordinating mediated interruptions: Social and system influence. In Proceedings of the 10th European Conference on Computer-Supported Cooperative Work. ESCW '07, pp. $351-370$.

Romero, N. A. and Markopoulos, P. (2009). Grounding interpersonal privacy in mediated settings. In Proceedings of the ACM 2009 international Conference on Supporting Group Work. GROUP '09. pp. 263-272.

Romero, N., Boer, L., and Markopoulos, P. (2009). Interactive and Lightweight Mechanisms to Coordinate Interpersonal Privacy in Mediated Communication. In Proceedings of the 12th IFIP TC 13 international Conference on HumanComputer interaction. Eds. Lecture Notes In Computer Science, vol. 5727, pp. 832833.

Roussopoulos, M., Maniatis, P., Swierk, E., Lai, K., Appenzeller, G., and Baker, M. (1999). Person-level routing in the mobile people architecture. In Proceedings of the second USENIX Symposium on Internet Technologies and Systems, USITS 1999

Wiberg, M. and Whittaker, S. (2005). Managing availability: supporting lightweight negotiations to handle interruptions. In ACM Transactions on Computer Human Interaction, 12 (4), pp. $356-387$.

Woodruff, A. and Aoki, P. M. (2003). How push-to-talk makes talk less pushy. In Proceedings of the 2003 international ACM SIGGROUP Conference on Supporting Group Work. GROUP '03. ACM, pp. 170 - 179. 